\title{
RESEARCH
}

Open Access

\section{Cord blood-endothelial colony forming cells are immunotolerated and participate at post-ischemic angiogenesis in an original dorsal chamber immunocompetent mouse model}

Richard Proust ${ }^{1 \dagger}$, Anne-Charlotte Ponsen ${ }^{1 \dagger}$, Valérie Rouffiac $^{2}$, Chantal Schenowitz ${ }^{3}$, Florent Montespan ${ }^{1}$, Karine Ser-Le Roux ${ }^{2}$, Frédéric De Leeuw² ${ }^{2}$ Corinne Laplace-Builhé ${ }^{2}$, Philippe Mauduit ${ }^{1}$, Edgardo D. Carosella ${ }^{3}$, Sébastien Banzet ${ }^{1}$, Jean-Jacques Lataillade ${ }^{1}$, Nathalie Rouas-Freiss ${ }^{3}$, Georges Uzan ${ }^{1+}$ and Juliette Peltzer ${ }^{1{ }^{* \dagger}}$ (D)

\begin{abstract}
Background: Cardiovascular diseases are the main cause of morbidity and mortality worldwide. Restoring blood supply to ischemic tissues is an essential goal for the successful treatment of these diseases. Growth factor or gene therapy efficacy remains controversial, but stem cell transplantation is emerging as an interesting approach to stimulate angiogenesis. Among the different stem cell populations, cord blood-endothelial progenitor cells (CBEPCS) and more particularly cord blood-endothelial progenitor cell-derived endothelial colony forming cells (CBECFCS) have a great proliferative potential without exhibiting signs of senescence. Even if it was already described that CB-ECFCs were able to restore blood perfusion in hind-limb ischemia in an immunodeficient mouse model, until now, the immunogenic potential of allogenic CB-ECFCs remains controversial. Therefore, our objectives were to evaluate the immune tolerance potency of CB-ECFCs and their capacity to restore a functional vascular network under ischemic condition in immunocompetent mice.
\end{abstract}

Methods: In vitro, the expression and secretion of immunoregulatory markers (HLA-G, IL-10, and TGF- $\beta 1$ ) were evaluated on CB-ECFCs. Moreover, CB-ECFCs were co-cultured with activated peripheral blood mononuclear cells (PBMCs) for 6 days. PBMC proliferation was evaluated by [3H]-thymidine incorporation on the last $18 \mathrm{~h}$. In vivo, CBECFCs were administered in the spleen and muscle of immunocompetent mice. Tissues were collected at day 14 after surgery. Finally, CB-ECFCs were injected intradermally in C57BL/6JRj mice close to ischemic macrovessel induced by thermal cauterization. Mice recovered until day 5 and were imaged, twice a week until day 30.

(Continued on next page)

\footnotetext{
* Correspondence: juliette.peltzer@inserm.fr

${ }^{\dagger}$ Richard Proust, Anne-Charlotte Ponsen, Georges Uzan and Juliette Peltzer contributed equally to this work.

'INSERM UMR-S-MD 1197/Ministry of the Armed Forces, Biomedical Research Institut of the Armed Forces (IRBA), Paul-Brousse Hospital Villejuif and CTSA Clamart, Clamart, France

Full list of author information is available at the end of the article
}

C C The Author(s). 2020 Open Access This article is licensed under a Creative Commons Attribution 4.0 International License, which permits use, sharing, adaptation, distribution and reproduction in any medium or format, as long as you give appropriate credit to the original author(s) and the source, provide a link to the Creative Commons licence, and indicate if changes were made. The images or other third party material in this article are included in the article's Creative Commons licence, unless indicated otherwise in a credit line to the material. If material is not included in the article's Creative Commons licence and your intended use is not permitted by statutory regulation or exceeds the permitted use, you will need to obtain permission directly from the copyright holder. To view a copy of this licence, visit http://creativecommons.org/licenses/by/4.0/ The Creative Commons Public Domain Dedication waiver (http://creativecommons.org/publicdomain/zero/1.0/) applies to the data made available in this article, unless otherwise stated in a credit line to the data. 
(Continued from previous page)

Results: Firstly, we demonstrated that CB-ECFCs expressed HLA-G, IL-10, and TGF- $\beta 1$ and secreted IL-10 and TGF- $\beta 1$ and that they could display immunosuppressive properties in vitro. Secondly, we showed that CB-ECFCs could be tolerated until 14 days in immunocompetent mice. Thirdly, we revealed in an original ischemic model of dorsal chamber that CB-ECFCs were integrated in a new functional vascular network.

Conclusion: These results open up new perspectives about using CB-ECFCs as an allogeneic cell therapy product and gives new impulse to the treatment of cardiovascular diseases.

Keywords: Endothelial progenitor cells, Cord blood-endothelial colony forming cells, Immunotolerance, Ischemia, Angiogenesis

\section{Background}

Cardiovascular diseases are the main cause of morbidity and mortality worldwide [1-3]. Restoring blood supply to ischemic tissues is an essential goal for a successful treatment of these diseases [4]. Several therapies based on delivery of pro-angiogenic factors, like VEGF, HGF, bFGF, or HIF-1, were considered as attractive treatment options to restore vascular network within ischemic tissues $[5,6]$. Growth factor therapy consists in delivering corresponding recombinant proteins by intra-arterial administration to stimulate angiogenesis. Although preclinical studies were promising, larger randomized clinical trials failed to demonstrate significant benefits [4]. This gene therapy is based on delivery of genes encoding angiogenic growth factors by intramuscular plasmid or viral vector injection. During the last two decades, many clinical trials assessed the efficacy and security of gene therapy products for critical limb ischemia. However, the effectiveness of gene therapy using pro-angiogenic factors remains controversial, and only further largescale clinical trials will clarify their efficacy [7-9].

Recently, cell transplantation-based therapy using stem or progenitor cells from different sources into ischemic tissues emerged as a new approach to stimulate angiogenesis and/or vasculogenesis. Among the different cell populations used, endothelial progenitor cells (EPCs), mesenchymal stromal cells (MSCs), and bone marrowmononuclear cells demonstrated their benefit in preclinical studies for ischemic disease treatment [10-12].

EPCs were discovered by Asahara in 1997 and include different cell progenitors with heterogeneous lineage origin and functions $[13,14]$. In vitro, mainly two distinct cell types have been identified [15]: (i) early EPCs or colony forming unit-endothelial cells (CFU-ECs) with myeloid phenotype displaying, in vivo, a paracrine angiogenic effect [16] and (ii) late EPCs or endothelial colony forming cells (ECFCs), also known as outgrowth endothelial cells that are the only progenitors with the ability to migrate to ischemic site and directly incorporate into new vessels during vascular network formation in vivo $[14,17,18]$. ECFCs could be isolated from different sources: bone marrow [19, 20], adult peripheral blood (APB) [13] or embryonic annexes like the umbilical cord blood [14, 17], umbilical cord [21], or more recently term placenta $[22,23]$.

Autologous APB-ECFCs appeared as the ideal candidates for cell therapy because of no risk of immunological rejection. However, when isolated from patients with vascular diseases, their low number and poor expansion properties limit their use as a cell therapy product.

Comparative studies demonstrated that CB-ECFCs are more clonogenic and have a greater proliferative potential without exhibiting signs of senescence $[14,18]$. Moreover, we demonstrated that the immaturity, more preserved in CB-ECFCs than in APB-ECFCs, is an essential prerequisite for vascular repair functionality [24]. Indeed, Au et al. demonstrated that in vivo co-implantation of CB-ECFCs together with pericytes in collagen gel implanted into cranial windows in SCID mice led to long-lasting and functional vascular network, while APB-ECFC-derived blood vessels were transient and almost completely disappeared in 21 days [17]. Finally, we and others have demonstrated that CB-ECFCs restore blood perfusion in hind-limb ischemia performed in an immunodeficient mouse model $[25,26]$. For all these reasons, despite a potential alloantigenic reaction from the host, CB-ECFCs seem to be the best valuable therapeutic tool. However, the nonimmunogenic potential of allogeneic CB-ECFCs remains controversial. Some studies reported that CB-ECFCs display low immunogenic features $[27,28]$. Finally, we have demonstrated in vitro that human CB-ECFCs could exert an immunosuppressive effect on mouse lymphocyte proliferation more accentuated than APB-ECFCs. Others stated that this low immunogenic state of ECFCs could only be obtained by co-culture with mesenchymal stromal cells [2, 3, 29]. To our knowledge, only Teofili's group used CB-ECFCs in a hind-limb ischemia model in immunocompetent mice, suggesting the immune tolerance of CB-ECFCs. These authors demonstrated that human CB-ECFCs improve blood flow recovery and restrain the damaging ischemia effect. However, the physical incorporation of human CB-ECFCs in the mouse vascular network was not clearly demonstrated [30]. 
Given the therapeutic interest of CB-ECFCs and the limited literature data concerning their immunogenicity, our study aims to verify their persistence in an allogeneic environment and to follow over time their ability to participate in the repair of a vascular network in an innovative model in immunocompetent animals.

In the present study, we demonstrated that CB-ECFCs express immunoregulatory markers like HLA-G, IL-10, and TGF- $\beta 1$, which are hypoimmunogenic and display immunosuppressive properties in vitro in a mixed lymphocyte reaction assay. We confirmed in vivo these tolerogenic features since human CB-ECFCs were still present 14 days after injection in the spleen and muscle of immunocompetent mice. Finally, using an original model of a dorsal chamber model implanted in immunocompetent mice, we proved that CB-ECFCs were not only tolerated but are also functional, since they directly integrated in a new functional vascular network under ischemic conditions.

\section{Methods}

\section{Endothelial cell (EC) isolation and culture}

Human umbilical cord blood samples were collected from healthy full-term newborns after signed maternal informed consent. According to the French law, article L.1243-3 of the Public Health Code, prior approval by an Institutional Review Board was not required. Samples were obtained through a partnership with the Cord Blood Bank of St Louis Hospital which is authorized by the National Agency for the Safety of Medicines and Health Products (ANSM; authorization no. PPC51). CBECFCs were isolated from cord blood mononuclear cells (CBMCs) collected by Ficoll (PAN-Biotech, Dutscher) density gradient centrifugation and were resuspended in a EGM-2-MV medium (Lonza, Levallois) as previously described [31, 32]. Briefly, CBMCs were then seeded in 12 -well culture plates coated with $50 \mu \mathrm{g} / \mathrm{mL}$ rat tail collagen type I (Corning, Boulogne-Billancourt) and cultured at $37^{\circ} \mathrm{C}$, under $5 \% \mathrm{CO}_{2}$, in a humidified incubator. After $24 \mathrm{~h}$ of culture, non-adherent cells and debris were aspirated. Adherent cells were washed once with PBS, and complete EGM-2-MV medium was added to each well. The medium was changed daily for 7 days and then every 2 days until the first passage. Colonies of endothelial cells appeared between 7 and 14 days of culture and were identified as well-circumscribed monolayers of cells with a cobblestone appearance. For all the experiments, CB-ECFCs were used at passages 3-5.

\section{Flow cytometry}

CB-ECFCs were detached with trypsin and immunolabeled with BD Cytofix/Cytoperm ${ }^{\text {Tx }}$ Fixation/ Permeabilization kit according to manufacturer's protocol (BD Biosciences). Briefly, CB-ECFCs were fixed and permeabilized in Fixation/Permeabilization solution for $20 \mathrm{~min}$ at $4{ }^{\circ} \mathrm{C}$. After 2 washings in BD Perm/Wash buffer, CB-ECFCs were immunolabeled with anti-soluble HLA-G5 (clone 2A12; Thermo Fisher Scientific), antiIL-10 (clone B-S10; Diaclone), or anti-TGF- $\beta 1$ (clone \#27235; R\&D Systems) antibodies for $30 \mathrm{~min}$ at $4{ }^{\circ} \mathrm{C}$. After 2 washings in BD Perm/Wash buffer, secondary antibody GAM-PE was incubated for $30 \mathrm{~min}$ at $4{ }^{\circ} \mathrm{C}$. After 2 washings in BD Perm/Wash buffer, data were acquired and analyzed on an Accuri C6 flow cytometer (BD Biosciences).

\section{CB-ECFC immunofluorescence staining}

CB-ECFCs were grown in coverslips, fixed in $4 \%$ paraformaldehyde for $10 \mathrm{~min}$ at $\mathrm{RT}$, rinsed twice in phosphate-buffered saline (PBS), and permeabilized in PBS/BSA $3 \% /$ Triton $0.1 \%$ for 5 min at RT. Soluble HLAG5 (clone 2A12), IL-10 (clone B-S10), TGF- $\beta 1$ (clone \# 27235), and CD31 immunostaining were performed for $1 \mathrm{~h}$ at RT. After 3 washings in PBS/BSA 3\%/Triton $0.1 \%$, CB-ECFCs were incubated with secondary antibodies goat anti-rabbit-Alexa Fluor 488 (Invitrogen) and donkey anti-mouse-Alexa Fluor 568 (Invitrogen) for $45 \mathrm{~min}$ at RT. After 3 washings in PBS/BSA 3\%/Triton $0.1 \%$, CBECFCs were then stained with $2 \mu \mathrm{g} / \mathrm{mL}$ of 40,6 -diamidino-2-phenylindole (DAPI) for $5 \mathrm{~min}$ at RT. After 3 washings in PBS $1 \times$, slides were embedded using Glycergel Mounting Medium (Dako). Images were acquired with the Leica SP5 confocal microscope (Leica Microsystems, Wetzlar, Germany) equipped with a $\times 63$ oil immersion fluorescence objective.

\section{ELISAs}

CB-ECFCs were grown for $24 \mathrm{~h}$ in EBM-2 supplemented with 5\% FBS. ELISAs against IL-10 and TGF- $\beta 1$ (R\&D Systems) were performed on supernatants according to manufacturer's instructions.

\section{Mixed lymphocyte reaction (MLR)}

CB-ECFCs were used as either stimulator (immunogenicity assay) or third-party cells (immunosuppression assay) toward HLA-mismatched peripheral blood mononuclear cells (PBMCs), as responder cells. PBMCs were isolated from the blood of healthy volunteer donors from the French Blood Establishment (EFS, Saint-Louis Hospital, Paris, France) after informed consent, by density gradient centrifugation over Ficoll-Paque PLUS (Sigma). Human B lymphoblastoid cell line (LCL) 721.221 (ATCC), HLA class II-positive cells, was irradiated at 75-Gy dose to be used as a stimulator in immunosuppression assay. The ratio of PBMCs and LCL seeded in each well of 96-well plate was 1:0.5, with a final concentration of $10^{5} \mathrm{PBMCs} /$ well. CB-ECFCs were used at various ratios (from $0.5 \times 10^{5}$ to $0.03 \times 10^{5}$ cells/ 
well). MLR lasted for 6 days at $37^{\circ} \mathrm{C}$ in a humidified $5 \%$ $\mathrm{CO}_{2}$ air atmosphere. On day $5,[3 \mathrm{H}]$-thymidine $(1 \mathrm{mCi} /$ well, PerkinElmer) was added to each well and incubated for another $18 \mathrm{~h}$. Cells were then harvested on filtermats $\mathrm{A}$, and thymidine incorporation into DNA was quantified using a $\beta$ counter (Wallac 1450; Pharmacia).

\section{CB-ECFC transduction}

At day 0, CB-ECFCs were incubated at 10,000 cells $/ \mathrm{cm}^{2}$ in EGM-2-MV medium (Lonza). At day 1, CB-ECFCs were infected with lentiviral vector containing the GFP (rLV.EF1.GFP, Vectalys) or mCherry (pEZ-Lv105, GeneCopoeia) transgene with $8 \mu \mathrm{g} / \mathrm{mL}$ polybrene (SIGMAAldrich) at a multiplicity of infection (MOI) of 5 for $24 \mathrm{~h}$ at $37^{\circ} \mathrm{C}$. After washing with PBS, CB-ECFCs were amplified and transduction efficiency was evaluated by flow cytometry analysis (Accuri).

\section{Animal}

All experimental procedures were performed in accordance with the European Community Council Directive (2010/63/UE) for the care and use of laboratory animals. Procedures on animals were authorized by the Ministère de l'Education Nationale, de l'Enseignement Supérieur et de la Recherche after approbation by the National Committee for Ethics in Animal Experimentation (CEEA No26; project 2017030813196126_v2).

\section{CB-ECFCs-GFP ${ }^{+}$intrasplenic and intramuscular administration}

After anesthesia by intraperitoneal injection of ketamine $(100 \mathrm{mg} / \mathrm{kg}) / x y l a s i n e ~(20 \mathrm{mg} / \mathrm{kg})$, eight-week-old male C57BL/6JRj mice were incised on their left lateral side to access to the spleen. Cells were injected intrasplenically into a single point. After wound suturing, a second injection of the same treatment was performed intramuscularly in the quadriceps. Sixteen C57BL/6JRj mice were divided into 2 groups (Gn): (G1) -8 control mice were injected with $40 \mu \mathrm{L}$ of PBS per injection, and (G2)-8 treated mice were injected with $1.10^{6} \mathrm{CB}$-ECFCs-GFP ${ }^{+}$ suspended in $40 \mu \mathrm{L}$ of PBS per injection. The spleen and quadriceps were collected at day 14 after surgery, embedded in Tissue-Tek OCT compound, frozen in liquid nitrogen, and kept at $-80^{\circ} \mathrm{C}$.

\section{Muscle and spleen immunofluorescence staining}

Frozen samples (muscle and spleen) were sectioned to $8 \mu \mathrm{m}$ at $-21^{\circ} \mathrm{C}$ using cryostat (Leica). Sections of the muscle and spleen were fixed in $4 \%$ paraformaldehyde for $10 \mathrm{~min}$ at $\mathrm{RT}$ and rinsed three times in PBS, and non-specific sites were saturated with PBS/NGS 5\% for $1 \mathrm{~h}$ at RT. Anti-human-CD31 immunostaining was performed for $1 \mathrm{~h}$ at RT. After 3 washings in PBS/NGS 5\%, muscle and spleen sections were incubated with secondary antibodies goat anti-mouse-Alexa Fluor 546 (Invitrogen) for $1 \mathrm{~h}$ at RT. After 3 washings in PBS/NGS $5 \%$, sections were then stained with $2 \mu \mathrm{g} / \mathrm{mL}$ of DAPI for $5 \mathrm{~min}$ at RT. After 3 washings in PBS, slides were embedded using Glycergel Mounting Medium (Dako). Images were acquired with a Leica SP5 confocal microscope (Leica Microsystems, Wetzlar, Germany) equipped with $\times 63$ oil immersion fluorescence objective.

\section{Dorsal chamber implantation and CB-ECFCs-mCherry ${ }^{+}$ injection}

Nine-week-old male C57BL/6JRj mice were weighed before anesthesia by gas inhalation (1.5\% isoflurane in 1.5-2 $\mathrm{L} / \mathrm{min}$ air flow). The surgical procedure was previously described in details [33]. Briefly, each mouse received a subcutaneous injection of $100 \mu \mathrm{L}$ of Lidocaïne $(21.33 \mathrm{mg} /$ $\mathrm{mL}$ ) in the scapular region before skin incision. A local antiseptic solution (Betadine ${ }^{\circ}$ ) was also applied. A skinfold was stretched keeping the dorsal median line at the top. The two faces of the dorsal chamber were positioned on each side of the skinfold and secured by sutures between two adjacent orifices. To limit the inflammatory reaction, a dermocorticoid ointment was spread on the side where the skin had not been removed. On the opposite side, the entire epidermis and upper dermis within the optical window was removed as close as possible to the edges. Cells were injected intradermally close to ischemia induced by thermal cauterization of a macrovessel $15 \mathrm{~min}$ before cell injection to allow sufficient heat dissipation for preventing cell injuries. Twelve C57BL/6JRj mice were included and divided into 3 groups (Gn): (G1) - 4 control mice injected with $20 \mu \mathrm{L}$ of PBS with vascular ischemia, (G2)-4 treated mice injected with 500,000 CB-ECFCs-mCherry ${ }^{+}$suspended in $20 \mu \mathrm{L}$ of PBS with ischemia, and (G3) -4 treated mice injected with 500,000 CB-ECFCs-mCherry ${ }^{+}$ suspended in $20 \mu \mathrm{L}$ of PBS without ischemia. The surgical procedure was ended by closing the dorsal chamber with glass coverslips maintained by rings sealed by mechanical pressure. Anesthetic gas was switched off, and Tolfedine $(4 \mathrm{mg} / \mathrm{kg}$ ) was subcutaneously injected before the animal woke. Mice recovered inside cages enriched with a cocoon and diet gel to facilitate food access while the animals regained their initial mobility. Mice were maintained for a maximum of 30 days after dorsal chamber implantation.

\section{Imaging procedure}

Mice recovered until day 5 and were imaged until day 30 twice a week onto a macroscope (AZ100M, Nikon) and once a week onto a confocal microscope (SP8, Leica) in wildfield and fluorescence mode after $100 \mu \mathrm{L}$ dextran $2000 \mathrm{kDa}$-FITC intravenous injection $(25 \mu \mathrm{g} / \mu \mathrm{L})$.

For macroscopic assessment, mice were placed in a lateral position on a heating table maintained at $37^{\circ} \mathrm{C}$, 
itself placed on the macroscope stage. Acquisitions were recorded with a $\times 1$ objective (numerical aperture (NA), 0.1 ) with 1.3 zoom which provided an image of the whole dorsal chamber in a single frame in bright field and fluorescence. Dextran 2000-kDa-FITC signal was detected using a GFP filter (emission (em), 515-550 nm bandwidth), while CB-ECFCs-mCherry ${ }^{+}$were observed with a TexasRed filter (em, 573-613 nm bandwidth).

Following macroscopic imaging, mice were examined in confocal microscopy. To prevent mice from developing hyperthermia and dehydration during extended acquisitions, the confocal microscope was enclosed in an incubator $\left(\mathrm{OkoLab}^{\circ}\right)$ connected to a control unit monitoring air flow, heating, and humidity. The dorsal chamber was mechanically locked unto a dedicated support adapted to the microscope stage, thus preventing image degradation due to cardiac and respiratory movements. Confocal acquisitions were performed with $\mathrm{a} \times 10$ (N.A. 0.40 , air objective) and $\times 25$ (N.A. 0.95 , water objective) objective lens. Scanning speed was set at $600 \mathrm{~Hz}$ using the bi-directional acquisition mode, for a $1024 \times 1024$ pixel or image and a Z-step between 8 and $12 \mu \mathrm{m}$. A mosaicking technique was required to scan CB-ECFCs and ischemia on a same image. Moreover, a slight frame average of 2 was applied to deblur image. The 488-nm and 552-nm laser powers for the green and red illumination were set at $5 \%$ of their maximal power in most cases $(25 \mathrm{~mW}$ and 20 $\mathrm{mW}$ respectively) and collected at $495-545 \mathrm{~nm}$ and at $562-622 \mathrm{~nm}$ respectively. We typically used these settings, but they could be adapted depending on fluorescent levels recorded at each time point.

\section{Statistical analysis}

The allostimulatory effect of CB-ECFCs on T cells was evaluated by comparing the mean of each donor's maximal stimulatory effect to the value $(100 \%)$ represented by the LCL (positive control) condition with the onesample, one-sided Wilcoxon test. The inhibitory effect of CB-ECFCs as a third party cell was evaluated by comparing to 0 the slope of a linear regression of the PBMC proliferation relative to the no-CB-ECFC condition $(100 \%)$ by the numeric level of the increasing CB-ECFC numbers ( 0 being no-CB-ECFC, 1 being 2500 CB-ECFC, and so on). Tests and graphical representations were carried out using stats, lmer, lmerTest, and ggplot2packages in $\mathrm{R}$ (3.4.1). Results are described as mean and bootstrapped $95 \%$ confidence interval.

IL-10 and TGF- $\beta 1$ quantifications were expressed as mean \pm SD. Prism 7.0 (GraphPad Software) was used to perform the Mann-Whitney test for evaluating the difference in cytokine concentration in supernatants. A two-tailed $p$ value $<0.05$ was considered significant.

\section{Results}

CB-ECFCs are hypoimmunogenic and exert immunosuppressive properties

CB-ECFCs are thought to have a large potential in therapies aimed at repairing vascular defects from various etiologies. In this context, our present work entailed assessing, from an immunological perspective, whether allogenic CB-ECFCs could be used without a risk of rejection instead of autologous CB-ECFCs. For this purpose, we evaluated both immunogenicity and immunosuppressive properties of CB-ECFCs in HLAmismatched settings.

To assess the immunogenicity of CB-ECFCs, we studied their ability to be recognized as allogeneic stimulating cells by HLA-mismatched PBMCs. Highly stimulatory HLA class II+ lymphoblastoid cell line (LCL) was used as a PBMC proliferation inducing control. Results show that PBMC alloproliferation means are significantly lower with CB-ECFCs (PBMC alloproliferation mean of 7.75\% [IC95 2.66-7.84]) compared to HLA class II+ LCL (PBMC alloproliferation mean of $100 \%)(" p<0.05)$.

To examine the immunosuppressive features of CBECFCs, we studied their ability to affect PBMC alloproliferation as third-party cells in a classical MLR. Results revealed that CB-ECFCs significantly inhibit PBMC alloproliferation in a dose-dependent manner using 4 distinct PBMC to CB-ECFC allogeneic combinations (Fig. 1a). The slope of a linear regression of PBMC alloproliferation (relative to the no-CB-ECFC control), by CB-ECFC dose number, was significantly below $0\left(^{*} p<\right.$ 0.05) (slope - 20.72 [IC95, - 21.62 to -19.9$]$ ) indicating that CB-ECFCs exert a dose-dependent inhibitory effect on PBMC alloproliferation using 6 distinct PBMC to CB-ECFC allogenic combinations (Fig. 1b).

\section{CB-ECFCs express immunosuppressive markers HLA-G, IL-10, and TGF- $\beta 1$}

Some previous works evaluated the immunological potential of CB-ECFCs because of their protection against allospecific cellular immune response. However, the mechanisms that confer this protection (anti-inflammatory molecule secretion, cell-cell interaction, cytotoxicity ...) are not well understood. In our team, we have recently demonstrated that the TNF/TNFR2 signaling pathway is a key regulatory factor in CB-ECFC immunosuppressive effect. In this study, we checked the expression of different anti-inflammatory cytokines by flow cytometry. As shown in Fig. 2a, CB-ECFCs constitutively expressed the antiinflammatory cytokines IL-10 and TGF- $\beta 1$ as well as the immune checkpoint HLA-G [34]. These results were confirmed by confocal microscopy. CB-ECFCs expressed IL10 , TGF- $\beta 1$, and the soluble HLA-G5 isoform in small intracytoplasmic vesicles (Fig. 2b). Moreover, ELISA 


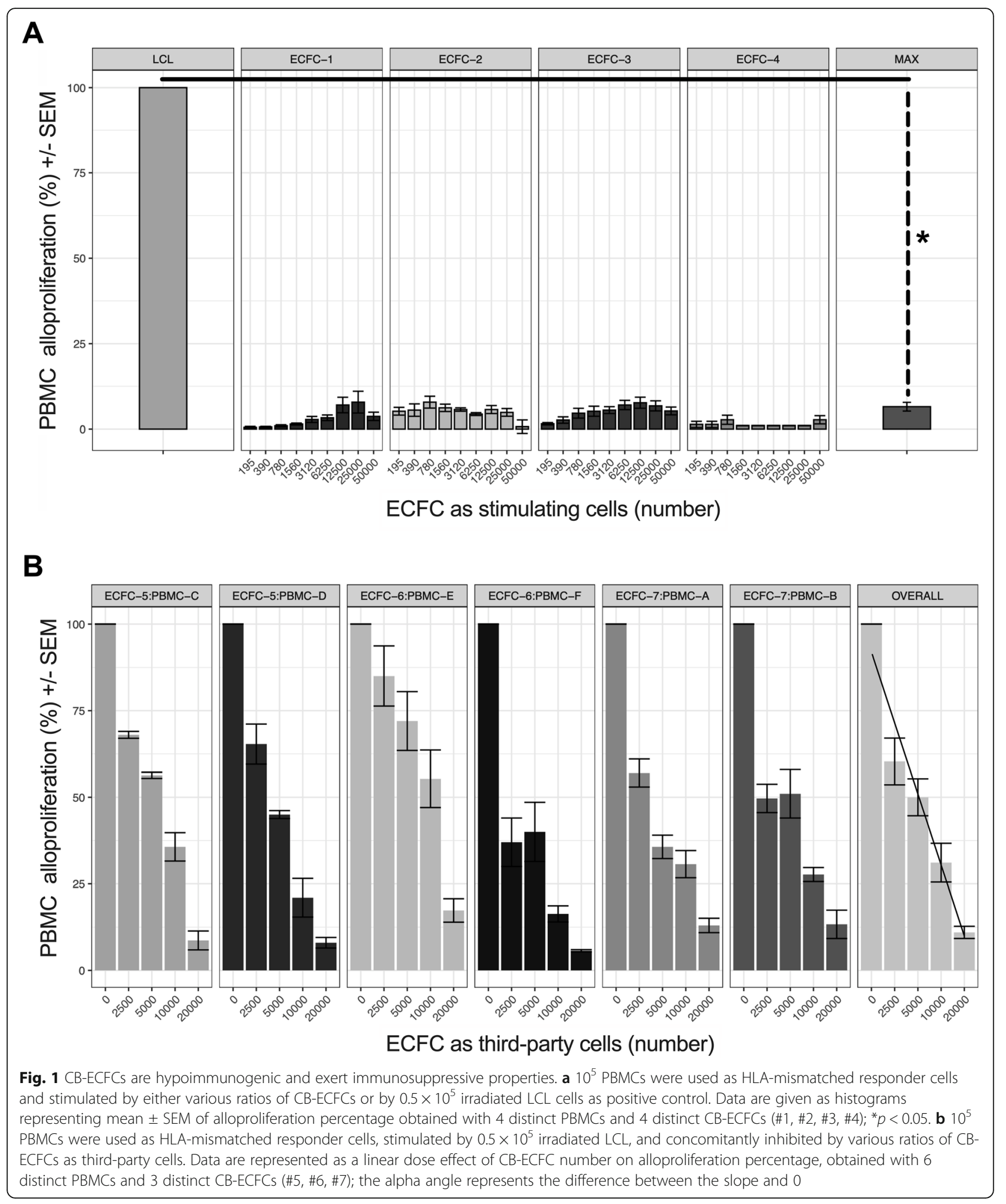

analysis of 5 distinct CB-ECFC supernatants showed that these cells were able to secrete IL-10 and TGF- $\beta 1$ ([IL$10]_{\mathrm{CB}-\mathrm{ECFCs}}=56.4 \mathrm{pg} / \mathrm{mL}$ and $[\mathrm{TGF}-\beta 1]_{\mathrm{CB}-\mathrm{ECFCs}}=633.7$ $\mathrm{pg} / \mathrm{mL} ;{ }^{* * *} p<0.01$, Fig. 2c, d).
Weibel-Palade bodies (WPB) are large vesicles specific to ECs and serve as a stock for von Willebrand factor (vWF) and P-selectin. Thereby, ECs contribute to hemostasis and inflammation by release of WPB. 

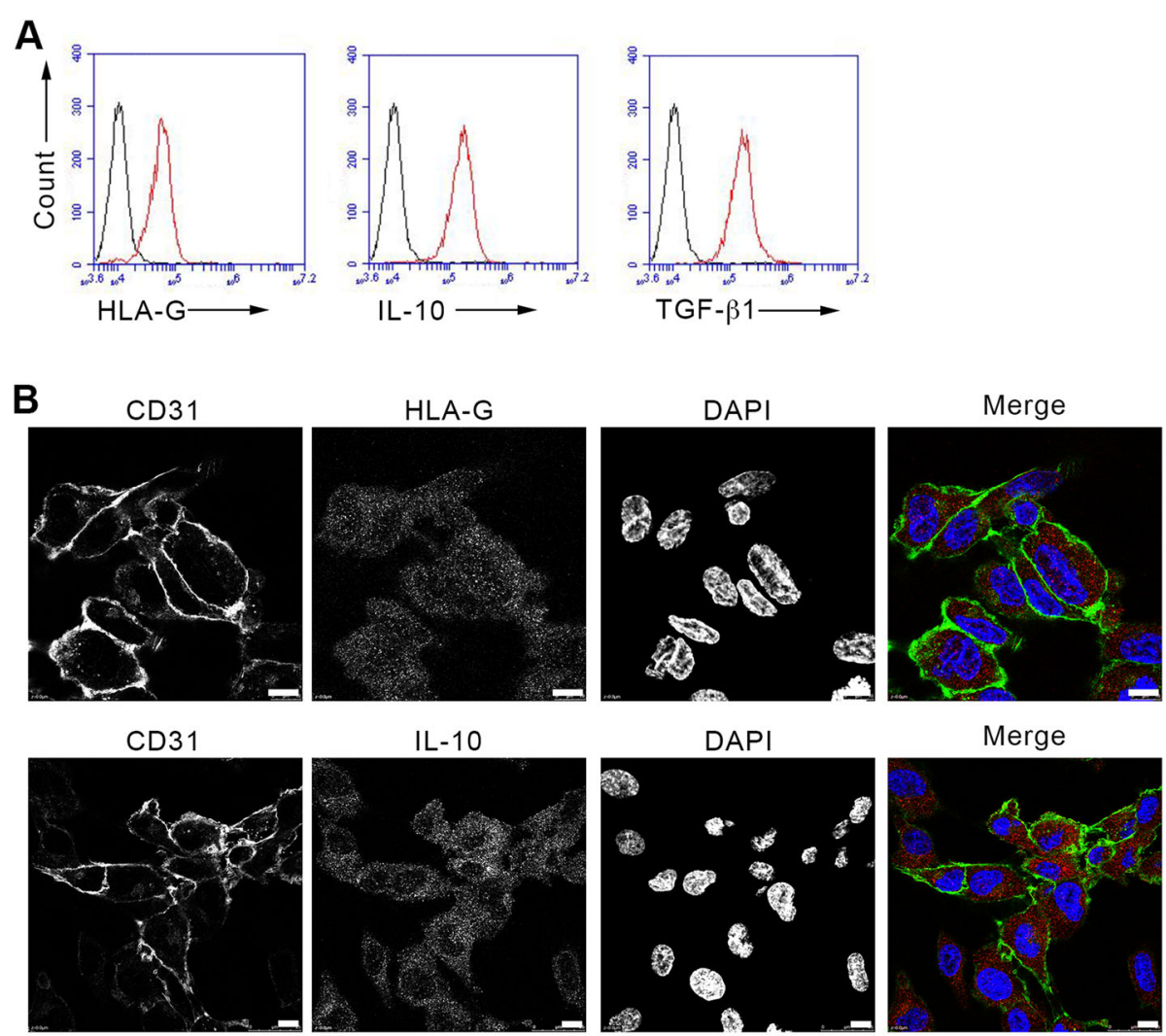

Merge

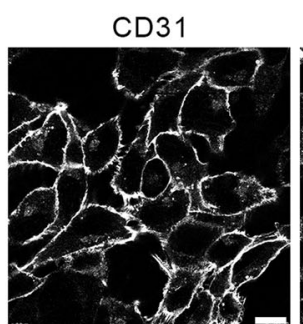

TGF- $\beta 1$
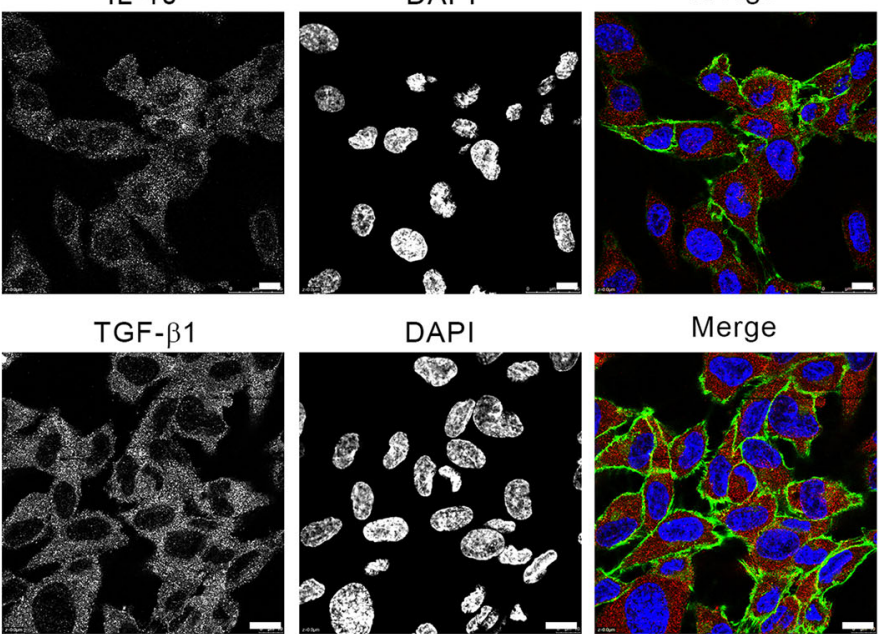

Merge

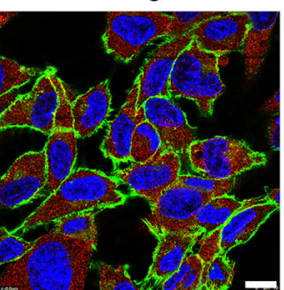

C

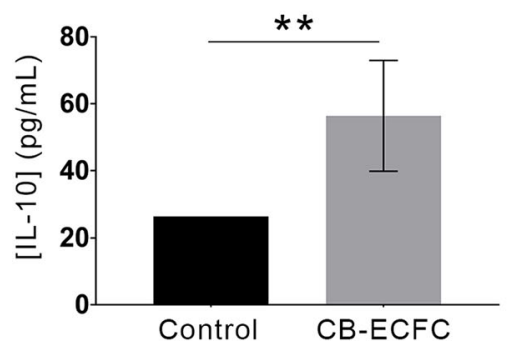

D

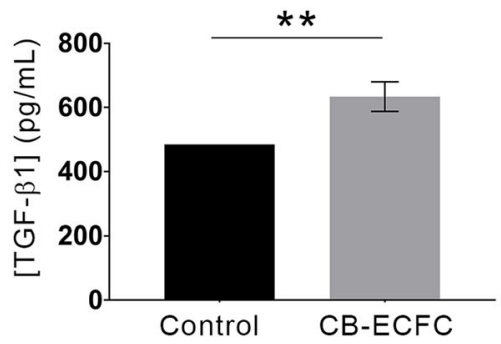

Fig. 2 CB-ECFCs express immunosuppressive markers HLA-G, IL-10, and TGF- $\beta 1$. a After fixation and permeabilization, CB-ECFCs were stained with anti-HLA-G5 (clone 2A12), anti-IL-10 (clone B-S10), and anti-TGF-B1 (clone \#27235) antibodies. Protein expressions were monitored and analyzed on a BD Accuri ${ }^{\mathrm{TM}}$ C6 flow cytometer. Data are mean fluorescence intensities (MFI); representative histograms of 3 experiments are shown (black curves, IgG isotype control; red curves, markers). b CB-ECFCs were grown on glass coverslips, fixed, permeabilized, and stained (left panels, CD31 staining; middle left panels: HLA-G5 or IL-10 or TGF- $\beta 1$ staining; middle right panels, DAPI staining; right panels, merges). Scale bars represent $10 \mu \mathrm{m}$. Representative images of 3 experiments are shown. c, d CB-ECFCs were grown for $24 \mathrm{~h}$ in EBM-2 supplemented with $5 \%$ FBS. Supernatants were collected and ELISAs were performed to quantify IL-10 (c) and TGF- $\beta 1$ (d). Data are given as histograms representing means \pm SD of 5 distinct CB-ECFCs; ${ }^{* *} p<0.01$; Mann-Whitney test 
However, using confocal microscopy, the antiinflammatory cytokines IL-10, TGF- $\beta$, and HLA-G were not co-localized with vWF, suggesting that these cytokines are not stocked in WPB (data not shown).

\section{CB-ECFCs are tolerated in in vivo immunocompetent environment}

To confirm the non-immunogenicity of CB-ECFCs in vivo, CB-ECFCs-GFP ${ }^{+}$were injected in the muscle and spleen of immunocompetent mice.

D14 after transplantation, the presence of CB-ECFCs$\mathrm{GFP}^{+}$was observed in the muscle and spleen by confocal microscopy. This observation is confirmed by the colocalization of CB-ECFCs-GFP ${ }^{+}$with specific humanCD31 immunostaining (Fig. 3).

\section{CB-ECFCs promote functional vascular network in immunocompetent mice}

To study the functionality of CB-ECFCs in vivo, we used an original immunocompetent mouse model consisting of dorsal chamber with macrovessel ischemia. Compared to hind-limb ischemia, the advantage of this model was to directly visualize the evolution of the vascularization on the same living mouse over time.

Macroscopic analysis revealed that the fluorescence of CB-ECFCs-mCherry ${ }^{+}$injected without macrovessel ischemia decreased over time and seemed to have almost disappeared at D26 (Fig. 4). This result was obtained using the same fluorescence exposure for the same mouse over time suggesting that the decrease in fluorescence over time, observed after injection of CB-ECFCs$\mathrm{mCherry}^{+}$without ischemia, corresponded to the death of these cells.
When CB-ECFCs-mCherry ${ }^{+}$were injected in the ischemic environment, the fluorescence signal was maintained up to 26 days after injection (Fig. 4).

When we focused in confocal microscopy on CBECFCs-mCherry ${ }^{+}$with ischemia condition at D26, vessels lined with mCherry cells were observed within the cluster of human cells injected. Moreover, when dextran-FITC was injected into the mouse vascular circulation, real-time green fluorescent flow was observed in vessels lined with CB-ECFCs-mCherry ${ }^{+}$(Fig. 5a), suggesting a connection between the mouse vascular circulation and human neo-vessels composed of human CBECFCs. More surprisingly, vessels lined with CB-ECFCs$\mathrm{mCherry}^{+}$were also founded at a distance from the human cell cluster (Fig. 5b).

\section{Discussion}

In vascular diseases such as critical limb ischemia, restoring blood supply is essential to effectively repair damaged ischemic tissues. Animal ischemic models show that CB-ECFCs injected into ischemic lesion actively participate to the formation of new vessels, leading to durable healing $[17,35]$. CB-ECFCs display high proliferative potential without obvious signs of senescence $[24,30]$; it is thus possible to reach the needed therapeutic dose with still highly active cells. Therefore, recent works promote the use of CB-ECFCs as a promising allogenic source for cell therapy in ischemic diseases; however, their immunological properties in allogeneic therapeutic use remain unclear.

In our study, we demonstrate that CB-ECFCs are nonimmunogenic and exert an immunosuppressive effect on PBMC response (HLA-mismatching). Indeed, we initially did not observe a significant alloproliferation of non-

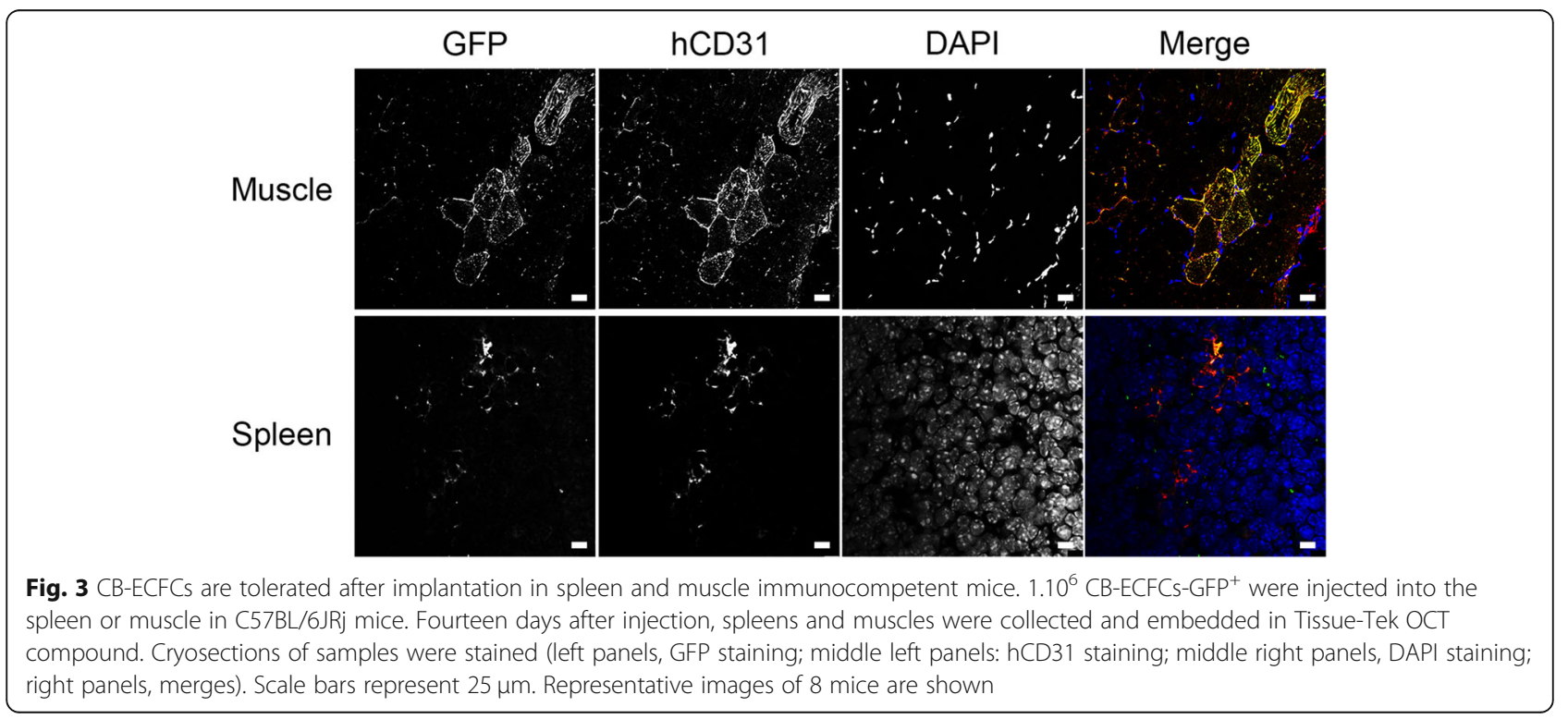




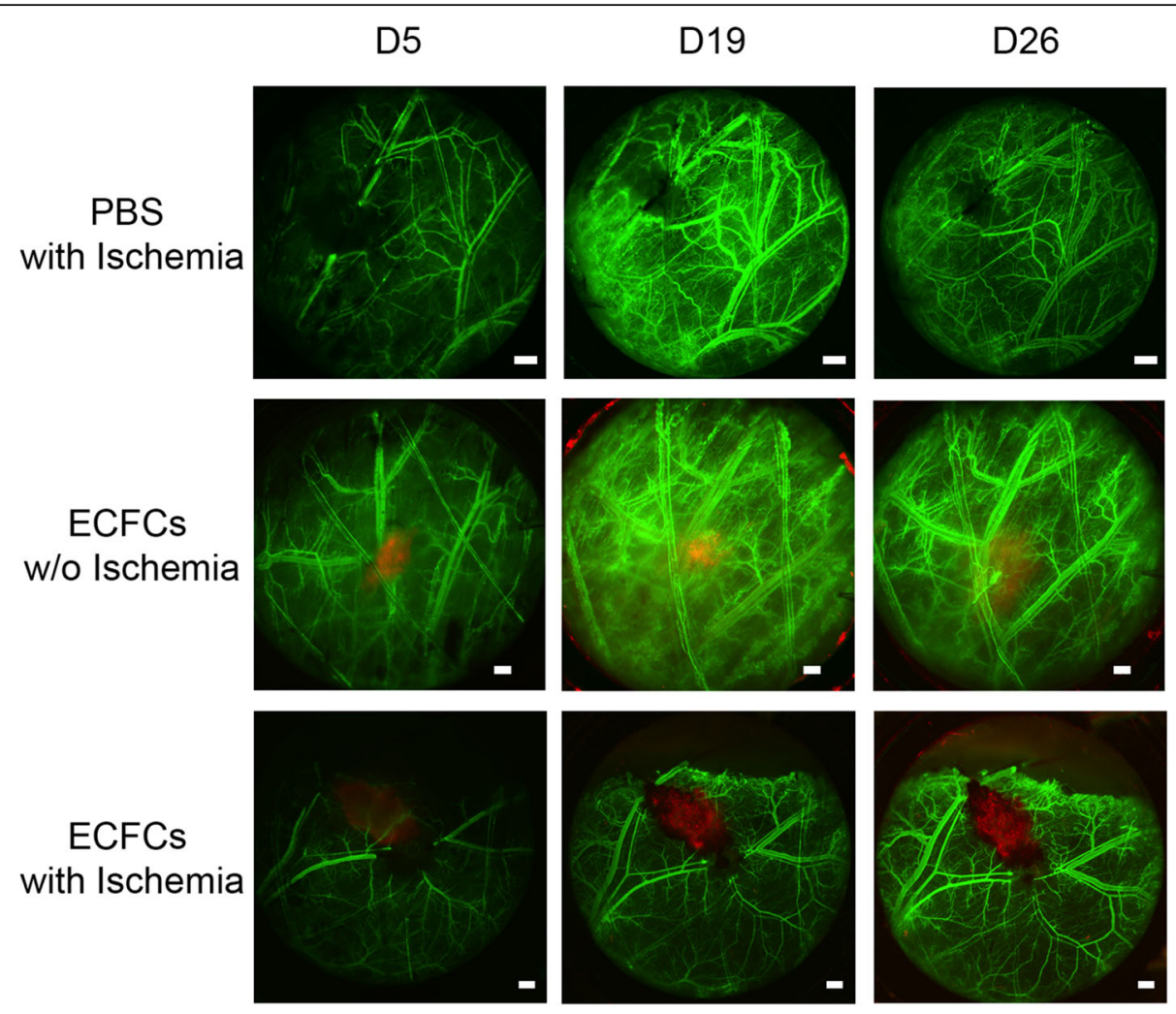

Fig. 4 CB-ECFCS survive in ischemic environment. Fifteen minutes after ischemia (top and bottom panels) induced by thermal cauterization, PBS (top panels) or $5.10^{5}$ CB-ECFCs-mCherry ${ }^{+}$(middle and bottom panels) were injected. Five, 19, and 26 days after treatment, dextran-FITC was injected by IV to reveal mouse vascular network. Scale bars represent $500 \mu \mathrm{m}$. Representative images of 4 mice per group are shown

activated PBMCs, suggesting their low ability to be recognized as allogenic stimulating cells by HLAmismatched PBMCs. In a second step, when CB-ECFCs were co-cultured with activated PBMCs, we observed a dose-dependent decrease in PBMC alloproliferation, suggesting an immunosuppressive capacity of these cells.

These results are in agreement with those obtained by Seifert and Pober groups who observed after stimulation with $\gamma$-IFN, a moderate proliferation of allogeneic CD4+ $\mathrm{T}$ cells cultured with EPC-derived ECs, demonstrating that these cells display a weak allostimulatory capacity against allogeneic CD4+ T cells in vitro [28, 36]. Control mature ECs from the same donor did not produce the same effect, indicating that this property is unique to EPC-derived ECs. Seifer et al. demonstrated in vivo that human CB-ECFCs transplanted in a xenogenic vascular graft model with decellularized aortic transplants did not trigger $\mathrm{T}$ cell infiltration and a weak infiltration of monocytes and macrophages suggesting that CB-ECFCs do not trigger any acute immune rejection. No intima remodeling was neither observed. In contrast, mature ECs triggered an immune response and massive inflammation [28]. To go further, our team demonstrated for the first time that the immunosuppressive effect of ECFCs is mediated by the TNF/TNFR2 signaling pathway.
Although several studies now tend to demonstrate that ECFCs display a certain level of non-immunogenicity, the underlying mechanisms are not yet fully understood. In this study, we hypothesized that the non-immunogenicity of CB-ECFCs could result from the expression of various anti-inflammatory molecules capable of protecting cells against the host's immune system. Indeed, we have demonstrated that CB-ECFCs contain in their cytoplasm the soluble HLA-G5 isoform, as well as IL-10 and TGF- $\beta$ molecules that are also be secreted by the cells.

Krishnamurthy et al. showed in a model of myocardial infarction in IL-10 KO mice treated or not with recombinant IL10 that EPC injected intramyocardially in the IL-10-treated group had a better survival. This was correlated with an improvement of angiogenesis and left ventricular function and the reduction of infarct size and fibrosis in the myocardium [37]. Moreover, the same team showed that IL-10 KO-EPC-derived exosomes inherit their parental dysfunctional phenotype. WT-EPCExo treatment enhanced endothelial cell proliferation and tube formation and inhibited apoptosis, whereas IL10 KO-EPC-Exo exhibited impaired or even detrimental effects [38]. TGF- $\beta$ also plays a key role in the angiogenic process of hypoxic tissue. For example, during stroke, neovascularization occurs primarily at the 

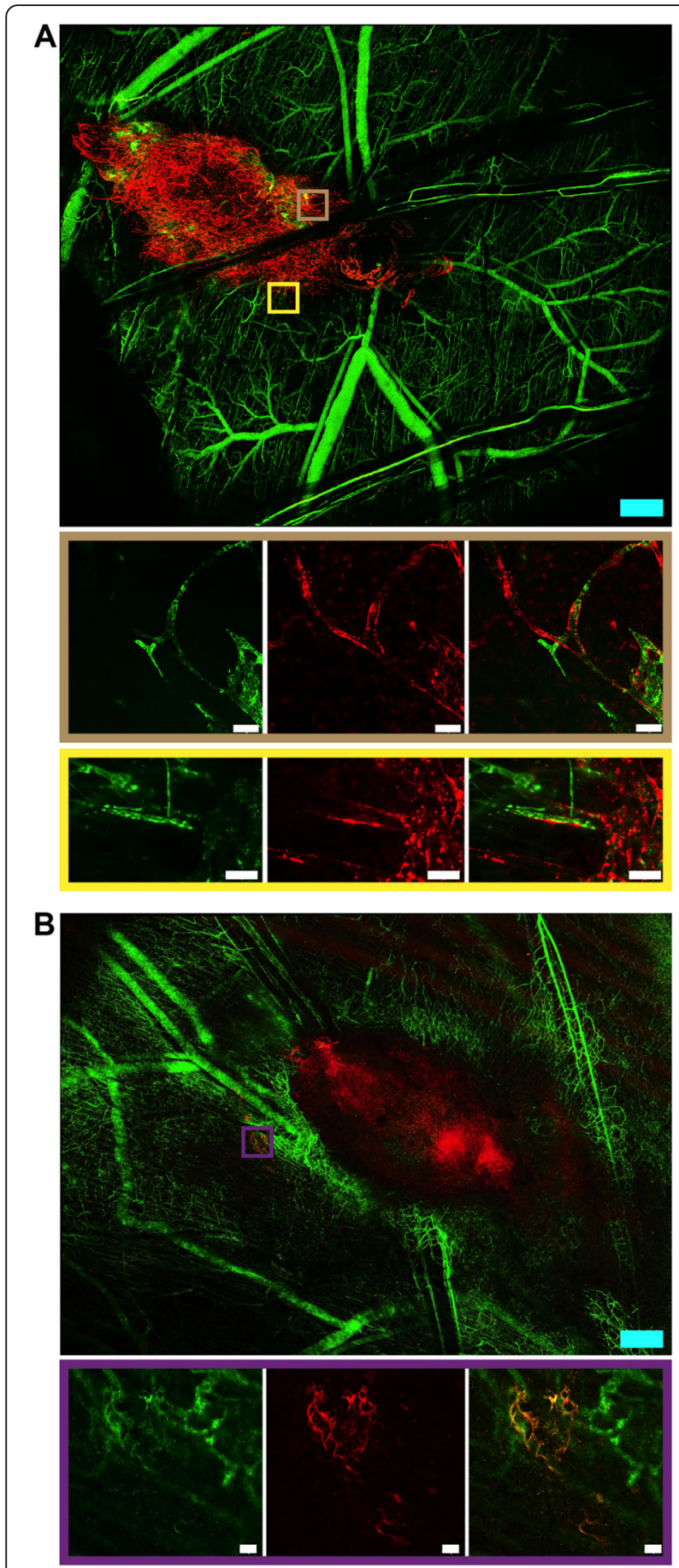

Fig. 5 CB-ECFCs promote functional vascular network in immunocompetent mice. Twenty-six days after ischemia, CB-ECFCs$\mathrm{mCherry}^{+}$structured themselves into functional vessels, integrated mouse vascular network as revealed by dextran-FITC flow inside at the site of injection (a) or in periphery to the site of injection (b). Blue and white scale bars respectively represent 500 and $50 \mu \mathrm{m}$. Representative images of 4 mice per group are shown periphery of the infarct, which correlates with high levels of both mRNA and active TGF- $\beta$ protein [39]. However, the expression levels could have contradictory effects. Indeed, low doses could promote angiogenesis and higher levels result in growth inhibition of endothelial cells and maturation of blood vessels [40, 41]. During immune injury, TGF- $\beta$ inhibits leukocyte adhesion and transmigration via inhibition of IL-8 and E-selectin expression, decreases cytokine-stimulated inducible nitric oxide synthase production, and increases endothelial nitric oxide synthase expression. Therefore, several TGF- $\beta$ actions on the endothelium during immune activation can be viewed as immunosuppressive [42]. Finally, HLAG, a non-classical HLA class I molecule, initially detected at the maternal-fetal interface, exerts inhibitory functions on cells responsible for graft rejection, without triggering an allogeneic response. Data of literature also indicated that soluble HLA-G5 isoform act on $\mathrm{T}$ cell proliferation by inhibiting the progression of alloreactive $\mathrm{T}$ cells [43]. Our results are consistent with those obtained by the Rizzo team in 2011 demonstrating that CD34+ progenitor cells derived from the cord blood express different isoforms of HLA-G molecules [44]. Based on this study, Nuzzolo et al. also hypothesized that the cytotoxic response against ECFCs could be reduced by low expression of HLA molecules and/or expression of immunoregulatory molecules such as HLA-G. In their study, the authors demonstrated that ECFCs from adult peripheral and cord blood, express HLA-ABC (class I) and HLA-DR antigens (class II) at levels comparable to those of MSCs, both before and after $\gamma$-IFN stimulation. Allogenic MSCs are commonly administered in vivo and do not trigger immune rejection. In addition, they demonstrated that CB-ECFCs were able to decrease the expression of mediators of inflammation and atherosclerosis produced by allogeneic lymphocytes and monocytes (IL$1 \beta$, IL-8, PGF, ALOX-5, TNF $\alpha$, CSF-2, MMP-1, MMP-9) more significantly than adult ECFCs [27].

In order to validate in vivo the non-immunogenic and immunosuppressive nature of CB-ECFCs, we first confirmed that these cells were not rejected after injection into the muscle and spleen of immunocompetent mice. Indeed, injected CB-ECFCs were still present 14days after injection in both tissues. Then, we used an original model of ischemia in mouse using a dorsal chamber to demonstrate that beyond their tolerance by the immune system of the recipient mouse, CB-ECFCs are able to form new vessels 26 days after transplantation. This new model was recently described in the literature to study the tumoral angiogenesis [33]. We adjusted this model to directly visualize angiogenesis after ischemia in a living mouse. These results are consistent with those obtained by the team of Teofili in a model of hind-limb ischemia in the immunocompetent mouse. Despite a 
progressive decline in the number of $\mathrm{hCD} 31^{+}$cells over time, a significant amount of human cells were still detected 28 days after transplantation. In addition, they observed that mice treated with CB-ECFCs showed an increase in microvascular density as compared to control without CB-ECFCs. Human CD31 ${ }^{+}$cells were detected inside vessels up to 4 weeks post-injection, suggesting the development of microvasculature from human origin. In their study, Flex et al. also showed a weak intramuscular inflammatory infiltration after transplantation of CB-ECFCs similar to that observed in our current study [30].

In another study from Shafiee et al., the authors observed that when ECFCs were transplanted in immunodeficient mice, the efficacy of transplantation and the number of new vessels formed were higher than they were in immunocompetent mice, suggesting that the immune system is an important parameter for ECFC transplantation [2]. However, the ECFCs used in this study were isolated from the placenta and it is now clearly admitted that the origin of the progenitor cells, including the ECFCs, can deeply influence their immunogenic phenotype. For example, we have previously demonstrated that cord blood MSCs are better tolerated than MSCs derived from the umbilical cord Wharton jelly or from the placenta (amnion or chorion) [45]. We have also demonstrated that the immunosuppressive effect was more accentuated in CB-ECFCs as compared to APB-ECFCs.

We demonstrated for the first time in an immunocompetent mouse model of ischemia that the vessels formed by CB-ECFCs 26 days after transplantation are directly connected to the blood circulation of the mouse. Indeed, after injecting dextran-FITC into the bloodstream, we can detect a flow within the vessels formed by the CB-ECFCs. These results are in agreement with several studies performed in different models of immunodeficient mice demonstrating the vasculogenic potential of ECFCs, with the formation of blood vessels generally observed 3 weeks after cell injections and maintained for at least 6 weeks $[17,29,35,36,46]$. These results also appear to correlate with the Teofili study performed in a model of lower limb ischemia in immunocompetent mice where they observed a significant increase in blood perfusion in the ischemic limb 28 days after intramuscular CB-ECFC transplantation [30].

\section{Conclusion}

Thus, through this study, we demonstrate for the first time that human CB-ECFCs, beyond their nonimmunogenicity and immunosuppressive capacity, are able to form functional blood vessels in immunocompetent mice, directly connected to the endogenous vascular network.
This proof of concept opens up perspectives about using CB-ECFCs as an allogeneic cell therapy product. It represents an interesting breakthrough and gives new impulse to the treatment of ischemic diseases. Allogenic CBECFCs are readily available through cord blood banks, and the ethical issue for their use is already well defined. They offer a robust cellular source for promoting vascular repair within the framework of tissue engineering.

\section{Abbreviations}

ALOX-5: Arachidonate 5-lipoxygenase; APB: Adult peripheral blood; bFGF: Basic fibroblast growth factor; CB: Cord blood; CBMCs: Cord blood mononuclear cells; CD: Cluster differentiation; CFU-ECs: Colony forming unitendothelial cells; CSF-2: Colony-stimulating factor-2; DAPI: 40,6-Diamidino-2phenylindole; DNA: Deoxyribonucleic acid; ECFCs: Endothelial colony forming cells; EGM-2-MV: Endothelial growth media-2-microvascular; EPCs: Endothelial progenitor cells; FITC: Fluorescein isothiocyanate; GFP: Green fluorescent protein; Gn: Groups; HGF: Hepatocyte growth factor; HIF-1: Hypoxia-inducible factor 1; HLA: Human leukocyte antigen; IFN: Interferon; IL: Interleukin; LCL: Lymphoblastoid cell line; MLR: Mixed lymphocyte reaction; MMP: Matrix metalloproteinase; MOI: Multiplicity of infection; MSCs: Mesenchymal stromal cells; PBMCs: Peripheral blood mononuclear cells; PBS: Phosphate-buffered saline; PE: Phycoerythrine; PGF: Placental growth factor; RT: Room temperature; TGF $\beta$ : Transforming growth factor- $\beta$; TNFa: Tumor necrosis factor-a; VEGF: Vascular endothelial growth factor; vWF: von Willebrand factor; WPB: Weibel-Palade bodies

\section{Acknowledgements}

The authors want to thank Dr. Cédric Thepenier for his help for the statistical analysis and UMS44 for technically and facility supports.

\section{Authors' contributions}

R.P. and A-C.P. designed and conducted the study, analyzed the data, and wrote the manuscript; V.R. designed and conducted the study and analyzed the data; C.S. and F.M. conducted the study and analyzed the data; K.S-L.R. designed and conducted the study; F.D.L. conducted the study; C.L-B. designed the study; P.M., E.D.C. and S.B. reviewed the manuscript; J-J.L., N.RF., and G.U. designed the study, analyzed the data, and reviewed the manuscript; J.P. designed the study, analyzed the data, and wrote the manuscript. The authors read and approved the final manuscript.

\section{Funding}

This work was supported by a grant from the Biomedical Research Institute of the Armed Forces PDH-1-SMO-1-0207.

\section{Availability of data and materials}

All data generated or analyzed during this study are included in this published article with the exception of IL-10, TGF- $\beta$, HLA-G, and VWF colocalized study. This data are available from anne-charlotte.ponsen@inserm.fr.

\section{Ethics approval and consent to participate}

All experimental procedures were performed in accordance with the European Community Council Directive (2010/63/UE) for the care and use of laboratory animals. Procedures on animals were authorized by the Ministère de l'Education Nationale, de l'Enseignement Supérieur et de la Recherche after approbation by the National Committee for Ethics in Animal Experimentation (CEEA №26; project 2017030813196126_v2).

\section{Consent for publication}

Not applicable.

\section{Competing interests}

The authors declare no competing interests.

\section{Author details}

${ }^{1}$ INSERM UMR-S-MD 1197/Ministry of the Armed Forces, Biomedical Research Institut of the Armed Forces (IRBA), Paul-Brousse Hospital Villejuif and CTSA Clamart, Clamart, France. ${ }^{2}$ Paris-Saclay University, Paris-Sud University, Gustave Roussy Institute, INSERM, CNRS, Molecular Analysis, Modeling and 
Imaging of Cancer Disease, Villejuif, France. ${ }^{3}$ CEA, DRF-IBFJ, Hemato-Immunology Research Unit, INSERM UMR-S 976, IRSL - Paris University, Saint-Louis Hospital, Paris, France.

Received: 15 February 2020 Revised: 30 March 2020 Accepted: 22 April 2020 Published online: 07 May 2020

\section{References}

1. Joseph P, Leong D, McKee M, Anand SS, Schwalm JD, Teo K, Mente A, Yusuf S. Reducing the global burden of cardiovascular disease, part 1: the epidemiology and risk factors. Circ Res. 2017;121(6):677-94.

2. Shafiee A, Patel J, Lee JS, Hutmacher DW, Fisk NM, Khosrotehrani K. Mesenchymal stem/stromal cells enhance engraftment, vasculogenic and pro-angiogenic activities of endothelial colony forming cells in immunocompetent hosts. Sci Rep. 2017:7(1):13558.

3. Shafiee A, Patel J, Wong HY, Donovan P, Hutmacher DW, Fisk NM Khosrotehrani K. Priming of endothelial colony-forming cells in a mesenchymal niche improves engraftment and vasculogenic potential by initiating mesenchymal transition orchestrated by NOTCH signaling. FASEB J. 2017:31(2):610-24

4. Deveza L, Choi J, Yang F. Therapeutic angiogenesis for treating cardiovascular diseases. Theranostics. 2012;2(8):801-14.

5. Carmeliet $P$, Jain RK. Molecular mechanisms and clinical applications of angiogenesis. Nature. 2011;473(7347):298-307.

6. Losordo DW, Dimmeler S. Therapeutic angiogenesis and vasculogenesis for ischemic disease. Part I: angiogenic cytokines. Circulation. 2004;109(21): 2487-91.

7. Sanada F, Taniyama Y, Azuma J, Yuka II, Kanbara Y, Iwabayashi M, Rakugi H, Morishita R. Therapeutic angiogenesis by gene therapy for critical limb ischemia: choice of biological agent. Immunol Endocr Metab Agents Med Chem. 2014;14(1):32-9.

8. Shimamura M, Nakagami H, Koriyama H, Morishita R. Gene therapy and cellbased therapies for therapeutic angiogenesis in peripheral artery disease. Biomed Res Int. 2013;2013:186215.

9. Tanaka M, Taketomi $\mathrm{K}$, Yonemitsu Y. Therapeutic angiogenesis: recent and future prospects of gene therapy in peripheral artery disease. Curr Gene Ther. 2014;14(4):300-8.

10. Osipova O, Saaya S, Karpenko A, Zakian S, Aboian E. Cell therapy of critical limb ischemia-problems and prospects. Vasa. 2019:48(6):461-71.

11. Qadura M, Terenzi DC, Verma S, Al-Omran M, Hess DA. Concise review: cell therapy for critical limb ischemia: an integrated review of preclinical and clinical studies. Stem Cells. 2018;36(2):161-71.

12. Ai M, Yan CF, Xia FC, Zhou SL, He J, Li CP. Safety and efficacy of cell-based therapy on critical limb ischemia: a meta-analysis. Cytotherapy. 2016;18(6): 712-24

13. Asahara T, Murohara T, Sullivan A, Silver M, van der Zee R, Li T, Witzenbichler B, Schatteman G, Isner JM. Isolation of putative progenitor endothelial cells for angiogenesis. Science. 1997;275(5302):964-7.

14. Ingram DA, Mead LE, Tanaka H, Meade V, Fenoglio A, Mortell K, Pollok K, Ferkowicz MJ, Gilley D, Yoder MC. Identification of a novel hierarchy of endothelial progenitor cells using human peripheral and umbilical cord blood. Blood. 2004;104(9):2752-60.

15. Medina RJ, Barber CL, Sabatier F, Dignat-George F, Melero-Martin JM, Khosrotehrani K, Ohneda O, Randi AM, Chan JKY, Yamaguchi T, et al. Endothelial progenitors: a consensus statement on nomenclature. Stem Cells Transl Med. 2017;6(5):1316-20

16. Mukai N, Akahori T, Komaki M, Li Q, Kanayasu-Toyoda T, Ishii-Watabe A, Kobayashi A, Yamaguchi T, Abe M, Amagasa T, et al. A comparison of the tube forming potentials of early and late endothelial progenitor cells. Exp Cell Res. 2008;314(3):430-40.

17. Au P, Daheron LM, Duda DG, Cohen KS, Tyrrell JA, Lanning RM, Fukumura D, Scadden DT, Jain RK. Differential in vivo potential of endothelial progenitor cells from human umbilical cord blood and adult peripheral blood to form functional long-lasting vessels. Blood. 2008;111(3):1302-5.

18. Bompais H, Chagraoui J, Canron X, Crisan M, Liu XH, Anjo A, Tolla-Le Port C, Leboeuf $M$, Charbord P, Bikfalvi $A$, et al. Human endothelial cells derived from circulating progenitors display specific functional properties compared with mature vessel wall endothelial cells. Blood. 2004;103(7):2577-84.

19. Guan XM, Cheng M, Li H, Cui XD, Li X, Wang YL, Sun JL, Zhang XY. Biological properties of bone marrow-derived early and late endothelial progenitor cells in different culture media. Mol Med Rep. 2013;8(6):1722-8.
20. Yu S, Li Z, Zhang W, Du Z, Liu K, Yang D, Gong S. Isolation and characterization of endothelial colony-forming cells from mononuclear cells of rat bone marrow. Exp Cell Res. 2018;370(1):116-26.

21. Zhang H, Tao Y, Ren S, Liu H, Zhou H, Hu J, Tang Y, Zhang B, Chen H. Isolation and characterization of human umbilical cord-derived endothelial colony-forming cells. Exp Ther Med. 2017;14(5):4160-6.

22. Patel J, Seppanen E, Chong MS, Yeo JS, Teo EY, Chan JK, Fisk NM, Khosrotehrani K. Prospective surface marker-based isolation and expansion of fetal endothelial colony-forming cells from human term placenta. Stem Cells Transl Med. 2013;2(11):839-47.

23. Rapp BM, Saadatzedeh MR, Ofstein RH, Bhavsar JR, Tempel ZS, Moreno O, Morone P, Booth DA, Traktuev DO, Dalsing MC, et al. Resident endothelial progenitor cells from human placenta have greater vasculogenic potential than circulating endothelial progenitor cells from umbilical cord blood. Cell Med. 2012;2(3):85-96.

24. Ferratge S, Ha G, Carpentier G, Arouche N, Bascetin R, Muller L, Germain S, Uzan G. Initial clonogenic potential of human endothelial progenitor cells is predictive of their further properties and establishes a functional hierarchy related to immaturity. Stem Cell Res. 2017:21:148-59.

25. Vanneaux V, El-Ayoubi F, Delmau C, Driancourt C, Lecourt S, Grelier A, Cras A, Cuccuini W, Soulier J, Lataillade JJ, et al. In vitro and in vivo analysis of endothelial progenitor cells from cryopreserved umbilical cord blood: are we ready for clinical application? Cell Transplant. 2010;19(9):1143-55.

26. Schwarz TM, Leicht SF, Radic T, Rodriguez-Arabaolaza I, Hermann PC, Berger F, Saif J, Bocker W, Ellwart JW, Aicher A, et al. Vascular incorporation of endothelial colony-forming cells is essential for functional recovery of murine ischemic tissue following cell therapy. Arterioscler Thromb Vasc Biol. 2012:32(2):e13-21

27. Nuzzolo ER, Capodimonti S, Martini M, lachininoto MG, Bianchi M, Cocomazzi A, Zini G, Leone G, Larocca LM, Teofili L. Adult and cord blood endothelial progenitor cells have different gene expression profiles and immunogenic potential. Blood Transfus. 2014;12(Suppl 1):s367-74.

28. Ladhoff J, Fleischer B, Hara Y, Volk HD, Seifert M. Immune privilege of endothelial cells differentiated from endothelial progenitor cells. Cardiovasc Res. 2010;88(1):121-9.

29. Souidi N, Stolk M, Rudeck J, Strunk D, Schallmoser K, Volk HD, Seifert M. Stromal cells act as guardians for endothelial progenitors by reducing their immunogenicity after co-transplantation. Stem Cells. 2017;35(5):1233-45.

30. Flex A, Biscetti F, lachininoto MG, Nuzzolo ER, Orlando N, Capodimonti S, Angelini F, Valentini CG, Bianchi M, Larocca LM, et al. Human cord blood endothelial progenitors promote post-ischemic angiogenesis in immunocompetent mouse model. Thromb Res. 2016;141:106-11.

31. Lavergne M, Vanneaux V, Delmau C, Gluckman E, Rodde-Astier I, Larghero J, Uzan G. Cord blood-circulating endothelial progenitors for treatment of vascular diseases. Cell Prolif. 2011;44(Suppl 1):44-7.

32. Chevalier F, Lavergne M, Negroni E, Ferratge S, Carpentier G, Gilbert-Sirieix M, Sineriz F, Uzan G, Albanese P. Glycosaminoglycan mimetic improves enrichment and cell functions of human endothelial progenitor cel colonies. Stem Cell Res. 2014:12(3):703-15.

33. Rouffiac V, Roux KS, Salome-Desnoulez S, Leguerney I, Ginefri JC, Sebrie C, Jourdain L, Lecluse $Y$, Laplace-Builhe C. Multimodal imaging for tumour characterization from micro- to macroscopic level using a newly developed dorsal chamber designed for long-term follow-up. J Biophotonics. 2020; 13(1):e201900217

34. Carosella ED, Rouas-Freiss N, Tronik-Le Roux D, Moreau P, LeMaoult J. HLAG: an immune checkpoint molecule. Adv Immunol. 2015;127:33-144.

35. Ding J, Zhao Z, Wang C, Wang CX, Li PC, Qian C, Teng GJ. Bioluminescence imaging of transplanted human endothelial colony-forming cells in an ischemic mouse model. Brain Res. 2016;1642:209-18.

36. Suarez Y, Shepherd BR, Rao DA, Pober JS. Alloimmunity to human endothelial cells derived from cord blood progenitors. J Immunol. 2007; 179(11):7488-96.

37. Krishnamurthy P, Thal M, Verma S, Hoxha E, Lambers E, Ramirez V, Qin G, Losordo D, Kishore R. Interleukin-10 deficiency impairs bone marrowderived endothelial progenitor cell survival and function in ischemic myocardium. Circ Res. 2011:109(11):1280-9.

38. Yue Y, Garikipati VNS, Verma SK, Goukassian DA, Kishore R. Interleukin-10 deficiency impairs reparative properties of bone marrow-derived endothelial progenitor cell exosomes. Tissue Eng Part A. 2017;23(21-22):1241-50.

39. Krupinski J, Kumar P, Kumar S, Kaluza J. Increased expression of TGF-beta 1 in brain tissue after ischemic stroke in humans. Stroke. 1996;27(5):852-7. 
40. Hofer E, Schweighofer B. Signal transduction induced in endothelial cells by growth factor receptors involved in angiogenesis. Thromb Haemost. 2007; 97(3):355-63.

41. Li C, Guo B, Bernabeu C, Kumar S. Angiogenesis in breast cancer: the role of transforming growth factor beta and CD105. Microsc Res Tech. 2001;52(4): 437-49.

42. Pintavorn P, Ballermann BJ. TGF-beta and the endothelium during immune injury. Kidney Int. 1997;51(5):1401-12.

43. Rouas-Freiss N, Naji A, Durrbach A, Carosella ED. Tolerogenic functions of human leukocyte antigen G: from pregnancy to organ and cell transplantation. Transplantation. 2007;84(1 Suppl):S21-5.

44. Buzzi M, Alviano F, Campioni D, Stignani M, Melchiorri L, Rotola A, Tazzari P, Ricci F, Vaselli C, Terzi A, et al. Umbilical cord blood CD34(+)cell-derived progeny produces human leukocyte antigen-G molecules with immunomodulatory functions. Hum Immunol. 2012;73(2):150-5.

45. Peltzer J, Montespan F, Thepenier C, Boutin L, Uzan G, Rouas-Freiss N, Lataillade JJ. Heterogeneous functions of perinatal mesenchymal stromal cells require a preselection before their banking for clinical use. Stem Cells Dev. 2015;24(3):329-44.

46. Kang KT, Lin RZ, Kuppermann D, Melero-Martin JM, Bischoff J. Endothelial colony forming cells and mesenchymal progenitor cells form blood vessels and increase blood flow in ischemic muscle. Sci Rep. 2017;7(1):770.

\section{Publisher's Note}

Springer Nature remains neutral with regard to jurisdictional claims in published maps and institutional affiliations.

Ready to submit your research? Choose BMC and benefit from:

- fast, convenient online submission

- thorough peer review by experienced researchers in your field

- rapid publication on acceptance

- support for research data, including large and complex data types

- gold Open Access which fosters wider collaboration and increased citations

- maximum visibility for your research: over $100 \mathrm{M}$ website views per year

At $\mathrm{BMC}$, research is always in progress.

Learn more biomedcentral.com/submissions 